\title{
Prévision du transport solide total Evaluation de formules existantes
}

\author{
António Heleno Cardoso \\ Département de Génie Civil de l'Instituto Superior Técnico, \\ Université Technique de Lisbonne, Portugal
}

Graça Oliveira Neves

Département d’Hydraulique du Laboratório Nacional de Engenharia Civil,

Lisbonne, Portugal

\section{Introduction}

Un des principaux objectifs de la recherche dans le domaine du transport de sédiments dans les canaux à fond mobile est la définition des formules susceptibles de fournir le transport solide en fonction des caractéristiques de l'écoulement, du fluide et des sédiments. Deux voies principales d'analyses ont été suivies : I'une basée sur des principes fondamentaux de I'hydrodynamique: l'autre basée sur l'analyse dimensionnelle.

Normalement, on considère deux formes de transport solide : le transport solide par charriage et le transport solide en suspension. Les deux formes diffèrent en deux aspects essentiels : dans le premier cas, les particules se déplacent par roulement sur le fond et leur vitesse est inférieure à celle de l'écoulement ; dans le deuxième cas. les particules se déplacent au sein de l'écoulement avec une vitesse du même ordre de grandeur que celle du fluide. Il n'existe pas de délimitation nette entre ces deux formes de transport : il y a aussi des auteurs qui considèrent une troisième forme caractéristique intermédiaire. la saltation, où les particules se déplacent essentiellement par des bonds, tout en alternant le charriage et la suspension.
A leur tour, les formules de prévision du transport solide peuvent aussi être classées comme formules de transport solide par charriage, en suspension. ou total (en suspension et par charriage). Les formules les plus divulguées fournissent la concentration du matériau de fond transporté et ne prennent pas en compte les matériaux de lavage (wash-load). De plus, le transport solide. qui est uniquement considéré comme une fonction des caractéristiques locales de l'écoulement et des sédiments, est égal à la capacité de transport.

La première formule de transport solide a été proposée par DuBoys, en 1879. Par la suite, beaucoup d'autres sont apparues. Le grand nombre de formules disponibles est le symptôme d'absence de leur universalité. En effet, les prévisions de ces formules varient considérablement parce qu'elles ne sont valables que pour les conditions spécifiques qui ont servi de base à l'établissement de leurs coefficients empiriques.

La première étude d'évaluation de formules de transport de sédiments a été développée par JOHNSON, en 1939 (in GOMEZ et CHURCH, [8]), en comparant leurs résultats à des données de laboratoire. Par la suite, beaucoup d'autres études ont été réalisées, notamment au cours des vingt dernières années, par WHITE et al.|22|, par BROWN.

\section{Prediction of the total sediment load. Appraisal of existing formulas}

In this study, the precision of ten sediment transport formulas is evaluated by comparing their results with existing data which cover flow regimes that occur in Tejo (Tagus) and Mondego rivers in Portugal. Several selection criteria were applied, allowing us to conclude that the formulas of Karim and Kennedy 90 and Karim and Kennedy 81 produce the best results, leading to small errors and covering a high percentoge of observed situations, both when applied to the entire data set or when applied to more homogeneous sub-sets of the same data. 
LIE [5] et par Gomez et Church [8]. Ces derniers ont étudié le cas de rivières sur lits de graviers.

Il y a plusieurs limitations conceptuelles à ce genre d'études, notamment la superposition qui peut exister entre les données utilisées dans l'évaluation et celles qui ont été utilisées pour établir les formules. Le nombre réduit de données obtenues pour des conditions naturelles dans les fleuves joue aussi un rôle important dans l'absence d'évaluations rigoureuses.

II n'existe donc pas une ou un nombre réduit de formules acceptées comme universellement adéquates. De ce fait, il est d'une grande utilité pratique d'identifier les meilleures pour certaines conditions d'écoulement et caractéristiques des sédiments (voir Bouvard [4], BAKKER et al. [3]). Par conséquent, nous avons décidé d'évaluer l'applicabilité de quelques formules parmi les plus connues, dans un domaine du plan $Z, \operatorname{Fr}\left(Z=h / D_{50}\right.$; $F r$-nombre de Froude; $h$-hauteur d'eau; $D_{50}$-diamètre median du matériel du fond) qui couvre des situations identifiées dans des fleuves portugais, en utilisant des données dont la qualité a déjà été testée à plusieurs reprises. Une description détaillée de cette étude est offerte par NEvEs [13].

\section{Formules de prévision du transport solide analysées}

Les formules analysées ont été choisies en tenant compte, entre autres, de leur facilité d'application, étant donné que celles qui exigent un nombre de variables plus réduit permettent l'utilisation d'un plus grand nombre de données existantes. Les formules sélectionnées sont les suivantes (ordre chronologique) :

ENGELUND et HANSEN (1967);

GRAF (1968);

SHEN et HUNG (1971);

ACKERS et WHITE (1973);

YANG (1979);

RAJU et al. (1981);

BROWNLIE (1981);

KARIM et KENNEDY (1981);

VAN RIJN (1984);

KARIM et KENNEDY (1990).

Les formules comme celles de MEYER-PETER et MÚLLER, de EINSTEIN-BROWN ou de FRIJLINK (entre autres), très usitées au Portugal, n'ont pas été choisies car elles prévoient seulement le transport solide par charriage alors que les données existantes concernent le transport solide total.

Dans cet article, nous ne présentons pas la description des formules indiquées. Nous offrons un résumé des variables qu'elles comportent, les limitations intrinsèques qui leur sont associées ainsi que les références bibliographiques où les formules peuvent être trouvées (voir tabl. 1). Par contre, dans l'Annexe, nous présentons les formules les plus précises pour les données utilisées.

L'analyse du tableau 1 nous permet de conclure que les variables caractéristiques de l'écoulement sont pratiquement les mêmes $(V, h, J)$ pour toutes les formules. Le diamètre caractéristique utilisé est presque toujours le même $\left(D_{50}\right)$, mises à part les formules de ACKERS et White $\left(D_{35}\right)$ et de VAN Ruj $\left(D_{50}\right.$ et $\left.D_{90}\right)$.

\section{Caractérisation des données utilisées}

\subsection{Généralités}

Un des aspects importants de l'évaluation de l'applicabilité des formules de prévision du transport solide est la sélection des données de test utilisées. Ces données doivent être cohérentes avec les conditions hydrauliques et sédimentologiques couvertes par les formules, différentes des données utilisées lors de leur calibration, de bonne qualité et en nombre suffisant.

Dans cette étude, nous avons utilisé des données du «World Flume Data Compendium " (WFDC). Ces dernières, compilées pour la première fois par PETERSON et HOWELLS [16], comportaient 6606 enregistrements (ensemble des valeurs des variables qui permettent de caractériser un écoulement uniforme en équilibre). Chaque enregistrement consistait en des valeurs de dix variables hydrauliques obtenues pour des canaux de laboratoire et des fleuves. Ces données ont été publiées par plusieurs auteurs. Il faut remarquer que 219 des enregistrements correspondent à des mesures faites sur le fleuve Mondego. En 1982, le Delft Hydraulics Laboratory a publié une extension du WFDC, tout en introduisant de nouvelles données et de l'information concernant la granulométrie du matériau du fond et la célérité des formes de fond. CARDoso et BELo [6] ont élaboré une nouvelle extension et correction des données, en ajoutant 415 enregistrements, parmi lesquels 265 correspondent à des mesures faites dans les fleuves Tejo et Mondego au Portugal. C'est cette dernière version du WFDC (avec 7106 enregistrements) qui a été utilisée dans cette étude.

Toutes ces données concernent des écoulements uniformes avec du transport solide en équilibre, ce qui correspond bien à une des conditions de validité des formules sélectionnées.

Nous présentons, par la suite, une description des données sélectionnées; nous identifions les méthodes utilisées dans le calcul de quelques variables nécessaires à l'évaluation des différentes formules dont les valeurs ne figurent pas dans les enregistrements mentionnés.

\subsection{Sélection des données utilisées}

Dans cette étude nous avons, au départ (voir OLIVEIRA et CARDoso $\mid 141)$. divisé les 7106 enregistrements qui composent le WFDC en 25 sous-ensembles relativement homogènes du plan $Z, F r$. Le paramètre $Z$ est défini par $h / D_{50}$, c'est-à-dire l'inverse de la rugosité relative. Le plan $\mathrm{Z}, \mathrm{Fr}$ a été utilisé par PETERSON et BLENCH [15] et par GraF et al. [10].

Nous présentons dans le tableau 2 les valeurs limites de séparation des 25 sous-ensembles, la désignation de chacun, $A_{i} A_{j}$, et, entre parenthèses, le nombre d'enregistrements qui sont contenus dans chaque sous-ensemble comportant des données du Tejo et du Mondego.

Les 484 enregistrements obtenus dans les fleuves portugais sont distribués dans les sous-ensembles $A_{2} A_{3}$, $A_{2} A_{4}, A_{3} A_{3}$ et $A_{3} A_{4}$. Ces données ne sont toutefois pas assez nombreuses pour caractériser globalement le régime alluvionnaire des fleuves portugais. Elles représentent une bonne base de travail, renforcée par le fait que nous 
Tableau 1. - Résumé des aspects fondamentaux des formules de prévision du transport solide.

\begin{tabular}{|c|c|c|c|c|}
\hline Formules & $\begin{array}{l}\text { Caractéristiques } \\
\text { des sédiments }\end{array}$ & $\begin{array}{l}\text { Caractéristiques } \\
\text { de l'écoulement }\end{array}$ & Limitations & $\begin{array}{c}\text { Référence } \\
\text { bibliographique }\end{array}$ \\
\hline $\begin{array}{l}\text { Engelund } \\
\text { et Hansen }\end{array}$ & $\mathrm{D}_{50}, \gamma_{\mathrm{s}}$ & $\mathrm{V}, \mathrm{h}, \mathrm{J}$ & $\begin{array}{c}\mathrm{D}_{50}>0,15 \mathrm{~mm} ; \quad \sigma_{\mathrm{g}}>2 ; \text { pas } \\
\text { valable pour des lits avec des } \\
\text { rides }\end{array}$ & Shen [18] \\
\hline Graf & $\mathrm{D}_{50}, \gamma_{\mathrm{S}}$ & h, J & $\begin{array}{c}0,5<\mathrm{J}<2,5 \\
0,3<\mathrm{D}_{50}(\mathrm{~mm})<24,0\end{array}$ & Graf [9] \\
\hline Shen et Hung & $\mathrm{D}_{50}, \gamma_{\mathrm{S}}$ & $\mathrm{V}, \mathrm{h}, \mathrm{J}$ & - & Shen [18] \\
\hline Ackers et White & $\mathrm{D}_{35}, \gamma_{\mathrm{S}}$ & $\mathrm{V}, \mathrm{h}, \mathrm{J}$ & $\mathrm{Fr}<0,8$ & Ackers et White [1] \\
\hline Yang & $\mathrm{D}_{50}, \gamma_{\mathrm{S}}$ & $\mathrm{V}, \mathrm{h}, \mathrm{J}$ & - & Yang [23] \\
\hline Raju et al. & $\mathrm{D}_{50}, \gamma_{\mathrm{S}}$ & $\mathrm{V}, \mathrm{h}, \mathrm{J}$ & - & Raju et al. [7] \\
\hline Brownlie & $\mathrm{D}_{50}, \gamma_{\mathrm{S}}, \sigma_{\mathrm{g}}$ & $\mathrm{V}, \mathrm{h}, \mathrm{J}$ & $0,0003<\mathrm{J}<3,7$ & Brownlie [5] \\
\hline $\begin{array}{c}\text { Karim et } \\
\text { Kennedy } 81\end{array}$ & $\mathrm{D}_{50}, \gamma_{\mathrm{S}}$ & $\mathrm{V}, \mathrm{h}, \mathrm{J}$ & - & $\begin{array}{c}\text { Karim et Kennedy } \\
{[11]}\end{array}$ \\
\hline Van Rijn & $\mathrm{D}_{50}, \mathrm{D}_{90}, \gamma_{\mathrm{S}}$ & $\mathrm{V}, \mathrm{h}, \mathrm{J}$ & $0,2<\mathrm{D}_{50}(\mathrm{~mm})<2,0$ & Van Rijn $[19,20]$ \\
\hline $\begin{array}{c}\text { Karim et } \\
\text { Kennedy } 90\end{array}$ & $\mathrm{D}_{50}, \gamma_{\mathrm{S}}$ & $\mathrm{V}, \mathrm{h}, \mathrm{J}$ & - & $\begin{array}{c}\text { Karim et Kennedy } \\
{[11]}\end{array}$ \\
\hline
\end{tabular}

$\mathrm{D}_{\mathrm{n}}$ - diamètre du matériau de fond dont $\mathrm{n} \%$ en poids est plus fin; $\gamma_{\mathrm{s}}$ - poids volumique du matériau de fond; $\mathrm{V}$ - vitesse moyenne de l'écoulement; $\mathrm{h}$ - hauteur moyenne de l'écoulement; $\mathrm{J}$ - perte de charge unitaire; Fr - nombre de Froude de l'écoulement; $\sigma_{\mathrm{g}}$ - coefficient de gradation du matériau de fond.

Tableau 2. - Caractérisation des sous-ensembles du plan Z, Fr.

\begin{tabular}{|c|c|c|c|c|c|}
\hline $\mathrm{Fr}_{\mathrm{Fr}} \mathrm{Z}$ & $10^{0}<Z<10^{1}$ & $10^{1}<Z<10^{2}$ & $10^{2}<Z<10^{3}$ & $10^{3}<Z<10^{4}$ & $10^{4}<Z<10^{5}$ \\
\hline $10^{0,5}<\mathrm{Fr} \leq 10^{1,0}$ & $\mathrm{~A}_{5} \mathrm{~A}_{1}$ & $\mathrm{~A}_{5} \mathrm{~A}_{2}$ & $\mathrm{~A}_{5} \mathrm{~A}_{3}$ & $\mathrm{~A}_{5} \mathrm{~A}_{4}$ & $\mathrm{~A}_{5} \mathrm{~A}_{5}$ \\
\hline $10^{0,0}<\mathrm{Fr} \leq 10^{0,5}$ & $\mathrm{~A}_{4} \mathrm{~A}_{1}$ & $\mathrm{~A}_{4} \mathrm{~A}_{2}$ & $\mathrm{~A}_{4} \mathrm{~A}_{3}$ & $\mathrm{~A}_{4} \mathrm{~A}_{4}$ & $\mathrm{~A}_{4} \mathrm{~A}_{5}$ \\
\hline $10^{-0,5}<\mathrm{Fr} \leq 10^{0,0}$ & $\mathrm{~A}_{3} \mathrm{~A}_{1}$ & $\mathrm{~A}_{3} \mathrm{~A}_{2}$ & $\mathbf{A}_{3} \mathbf{A}_{3}$ (1559) & $\mathbf{A}_{\mathbf{3}} \mathbf{A}_{\mathbf{4}}$ & $\mathrm{A}_{3} \mathrm{~A}_{5}$ \\
\hline $10^{-1.0}<\mathrm{Fr} \leq 10^{-0.5}$ & $\mathrm{~A}_{2} \mathrm{~A}_{1}$ & $\mathrm{~A}_{2} \mathrm{~A}_{2}$ & $\mathbf{A}_{2} \mathbf{A}_{3}$ & $\mathbf{A}_{\mathbf{2}} \mathbf{A}_{\mathbf{4}}$ & $\mathrm{A}_{2} \mathrm{~A}_{5}$ \\
\hline $10^{-2,0}<\mathrm{Fr} \leq 10^{-1,0}$ & $A_{1} A_{1}$ & $A_{1} A_{2}$ & $A_{1} A_{3}$ & $\mathrm{~A}_{1} \mathrm{~A}_{4}$ & $A_{1} A_{5}$ \\
\hline
\end{tabular}


avons retenu toutes les données du WFDC pour des valeurs similaires de $Z$ et de $F r$, totalisant ainsi 2739 enregistrements. Nous avons organisé les données en deux groupes :

- Groupe 1, comportant la totalité des 2739 enregistrements :

- Groupe 2, concernant seulement les enregistrements obtenus dans les fleuves portugais.

\subsection{Caractérisation des données utilisées}

Suite à la contribution du Delft Hydraulics Laboratory, chaque enregistrement s'est trouvé composé de 24 variables, dont le débit, $Q$, la hauteur de l'écoulement, $h$, et la largeur de la section, $B$, sont toujours présents. Les valeurs de la perte de charge unitaire, $J$ (considérée égale à la pente de la ligne d'énergie), du coefficient de gradation du matériau de fond, $\sigma_{g}$, de la concentration des sédiments, $C$, de la température, $T$, du type de formes de fond, des diamètres caractéristiques des sédiments $\left(D_{16}, D_{36}, D_{\text {moyen }}, D_{50}, D_{65}, D_{84}\right.$ et $\left.D_{90}\right)$, de la vitesse de chute des particules, $\omega$, de la longueur, $\lambda$, et de la hauteur, $\Delta$, des aspérités de fond (dunes, rides, antidunes, etc.) et de leurs célérités, existent dans la plupart des enregistrements.

Pour étudier l'applicabilité des formules nous avons besoin des valeurs de différentes variables, en fonction des paramètres qu'elles considèrent. Nous avons, plus précisément, besoin des valeurs de $J$ et de $C$, de $Q, h$ et $B$, ainsi que de trois diamètres caractéristiques du matériau du fond. Toutes ces variables existent dans les enregistrements qui font l'objet de cette étude. De plus, elles satisfont toutes les exigences suivantes:

$-B / h>4$, pour ne pas avoir des effets de paroi ;

$-\sigma_{g}<5$, pour exclure les courbes granulométriques bimodales :

- $0,062 \mathrm{~mm}<D_{50}<4,0 \mathrm{~mm}$, pour ne considérer que du sable et du gravier fin ;

$-C>10 \mathrm{ppm}$, valeur limite au-dessous de laquelle nous admettons que les valeurs sont du même ordre de grandeur que les erreurs de mesure.

Pour appliquer quelques-unes des formules, nous avons besoin de valeurs des variables telles que la viscosité cinématique, $\nu$, le rayon hydraulique du fond, $R_{b}$ (rayon hydraulique qui peut être obtenu en éliminant les effets de paroi, c'est-à-dire, où l'on ne tient compte uniquement que de la rugosité du fond) et, dans les cas où cela n'est pas connu, de la vitesse de chute des particules, $\omega$. Le calcul de la viscosité cinématique de l'eau a été fait selon WEAST et ASTLE [21]; pour le calcul du rayon hydraulique du fond nous avons suivi la méthode de VANONI et BROOKS (ASCE [2], p. 153); la vitesse de chute des particules a été obtenue à partir de la formule de RUBEY (ASCE [2], p. 175).

D'après le tableau 3, le Groupe / contient 812 enregistrements concernant des données des fleuves $(\approx 30 \% \mathrm{du}$ total) et 1927 données de laboratoires; le Groupe 2 (Tejo et Mondego), contient environ $60 \%$ de toutes les données de fleuves existant dans le Groupe 1.

Nous pouvons constater sur la figure $l$ qu'une grande partie des données $(57 \%)$ se trouve dans une petite zone qui correspond au sous-ensemble $A_{3} A_{3}$. Les données des fleuves, notamment celles du Tejo et du Mondego, sont

Tableau 3. - Nombre d'enregistrements de chaque groupe et sa répartition selon le type de canal.

\begin{tabular}{|c|c|c|}
\hline \begin{tabular}{|ll} 
Type de données & Groupe \\
\end{tabular} & 1 & 2 \\
\hline Fleuve & 812 & 484 \\
\hline Laboratoire & 1927 & - \\
\hline Fleuves Portugais & 484 & 484 \\
\hline Total & 2739 & 484 \\
\hline
\end{tabular}

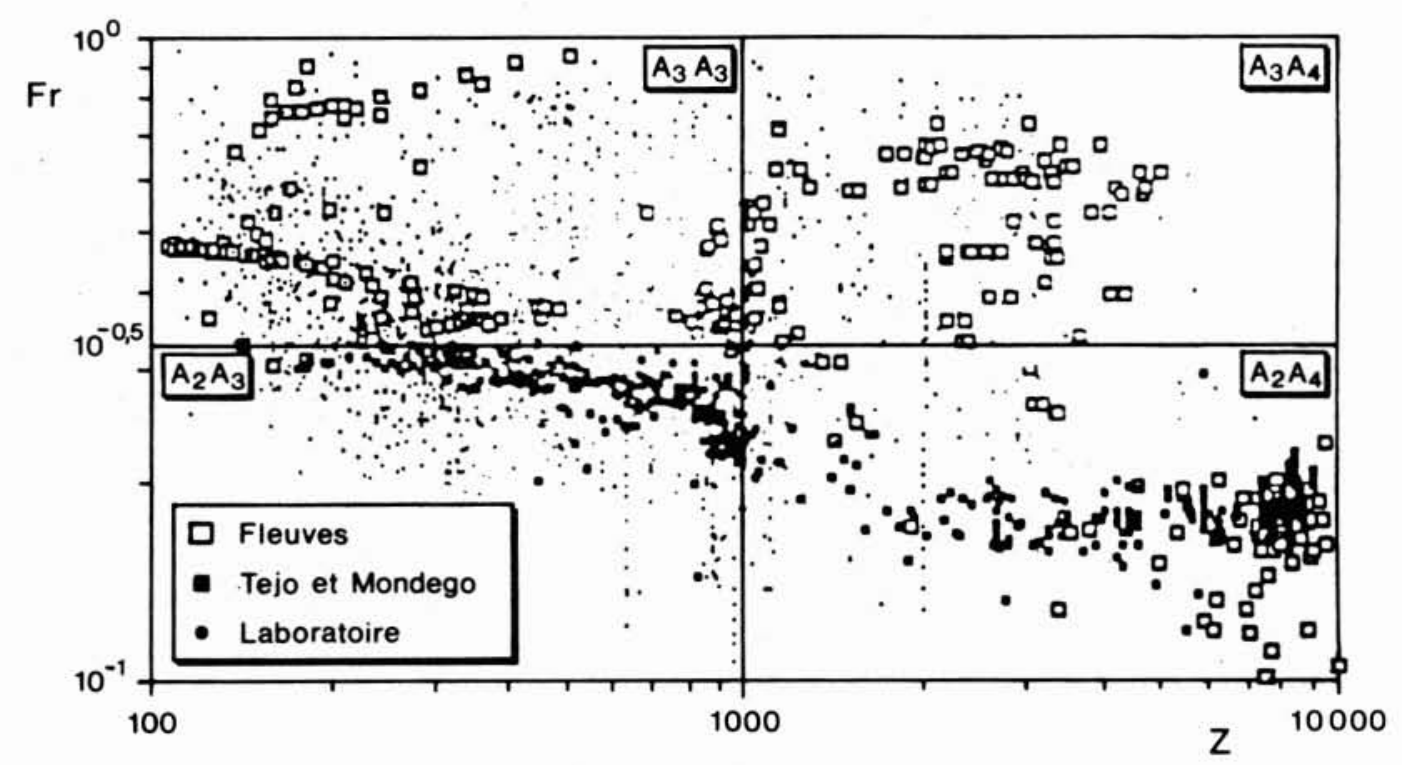

1. Répartition des données utilisées dans le plan Z, Fr. 
surtout réparties dans les sous-ensembles $A_{2} A_{3}$ et $A_{2} A_{4}$, c'est-à-dire qu'elles présentent des valeurs de nombres de Froude plus petits que $10^{-0.5}(\approx 0,32)$; les données de laboratoire se concentrent dans les sousensembles $A_{2} A_{3}$ et $A_{3} A_{3}$, c'est-à-dire que la majorité se caractérise par des valeurs de $Z$ plus petites que 2000 .

Le domaine de variation des principales variables est résumé dans le tableau 4 . Nous pouvons en conclure que les données des fleuves portugais ont, par comparaison avec les données du Groupe I, des caractéristiques plus homogènes : $F r$ varie entre 0,13 et 0.39 et $Z$ varie entre 175 et 8764 . Ces données présentent aussi de faibles valeurs de concentration du matériau solide ; dans $90 \%$ des cas cette variable est inférieure à $300 \mathrm{ppm}$. En ce qui concerne les valeurs de $D_{50}$ et de $\sigma_{g}$, nous pouvons encore constater une grande uniformité, ce qui est, en partie, une conséquence du fait que les données ont été obtenues dans des fleuves avec des lits de sable ou de graviers fins.

Tableau 4. - Domaine de variation des variables de chaque groupe.

\begin{tabular}{|c|c|c|}
\hline Variable & Groupe 1 & Groupe 2 \\
\hline $\mathrm{V}(\mathrm{m} / \mathrm{s})$ & 0,12 à 2,38 & 0,36 à 1,93 \\
$\mathrm{~h}(\mathrm{~m})$ & 0,02 à 6,66 & 0,40 à 6,66 \\
$\mathrm{~B}(\mathrm{~m})$ & 0,15 à 470,61 & 19,58 à 377,48 \\
$\mathrm{~J}\left(\times 10^{-3}\right)$ & 0,019 à 16,540 & 0,070 à 0,970 \\
$\mathrm{D}_{50}(\mathrm{~mm})$ & 0,007 à 7,00 & 0,73 à 2,60 \\
$\sigma_{\mathrm{g}}$ & 1,00 à 13,83 & 2,04 à 2,73 \\
$\mathrm{~B} / \mathrm{h}$ & 1,08 à 579,82 & 3,76 à 323,31 \\
$\mathrm{C}(\mathrm{ppm})$ & 0,0 à 98100,0 & 3,4 à 1166,0 \\
$\mathrm{Fr}$ & 0,11 à 1,00 & 0,13 à 0,39 \\
$\mathrm{Z}$ & 100,0 à 9955,5 & 174,5 à 8764,2 \\
\hline
\end{tabular}

\section{Evaluation des formules sélectionnées}

\subsection{Méthode d'évaluation}

L'évaluation des formules de prévision du transport solide a été faite en comparant les valeurs de la concentration des sédiments mesurée, $O i$, avec la concentration calculée. $\mathrm{C} i$. en ayant recours à leur rapport $\mathrm{Ci} / \mathrm{Oi}$. Ce dernier a aussi été utilisé par WHITE et al. [22] et par BROWNLIE [5].

Nous avons vérifié que ni la distribution normale ni le log-normal à deux paramètres ne s'adaptent à la distribution de $\mathrm{Ci} / \mathrm{Oi}$. Il s'ensuit que les critères d'évaluation qui se basent sur la valeur de la moyenne des $\mathrm{Ci} / \mathrm{Oi}$ ne sont pas valables car ils ont tendance à pénaliser excessivement les formules qui surestiment la concentration des sédiments $(\mathrm{Ci} / \mathrm{O} i>1)$. Si l'on tient compte du fait que, pour une formule adéquate, la moyenne de $\mathrm{Ci} / \mathrm{O} i$ doit être égale à 1, l'utilisation d'un critère basé sur la moyenne pénalise également une formule où cette moyenne vaut
0,5 et une autre où elle est égale à 1,5 , tandis que ces deux formules produisent des erreurs différentes, respectivement égales à $100 \%$ et à $50 \%$.

Par conséquent, les valeurs de la médiane (mesure de localisation) et des centiles 10 et 90 (mesures de dispersion), ainsi que le pourcentage des valeurs avec des erreurs inférieures à $100 \%$, soit par excès soit par défaut (c'est-à-dire, avec $1 / 2<C i / O i<2$ ), ont été adoptées pour caractériser l'ajustement de chaque formule aux valeurs mesurées. Un autre paramètre utilisé et qui traduit aussi l'ajustement a été le suivant :

$$
S=\sqrt{\frac{\Sigma\left[(\log O i-\log C i)^{2}\right]}{n-1}}
$$

où $n$ est le nombre de données. La valeur de $S$ est calculée pour les cas où le rapport $\mathrm{Ci} / \mathrm{Oi}$ est compris entre les centiles 10 et 90 ce qui, selon MURTEIRA [12], p. 87, n’a pas de conséquence importante sur la valeur de $S$.

\subsection{Evaluation des formules pour l'ensemble des données de chaque groupe}

\subsubsection{Données du Groupe I}

Les résultats de l'étude qui concernent le Groupe I (totalité des enregistrements) sont présentés dans le tableau 5 ainsi que sur la figure 2. Ils comprennent des valeurs de la médiane et des centiles 10 et 90 (de $\mathrm{Ci} / \mathrm{Oi}$ ), obtenues par application de chaque formule, du pourcentage des situations caractérisées par des erreurs inférieures à $100 \%(1 / 2<\mathrm{Ci} / \mathrm{O} i<2)$, de la valeur de $S$ et du pourcentage des données pour lesquelles une formule peut être appliquée (\% de données couvertes). Pour les trois formules qui produisent les meilleures prévisions, les histogrammes des fréquences de $\mathrm{Ci} / \mathrm{Oi}$, sous forme numérique. sont présentés dans le tableau 6.

Les résultats des formules sont très différents les uns des autres (voir, par exemple, les valeurs de la médiane et de $S$ dans le $t a b l .5$ et sur la fig. 2).

La formule de KARIM et KENNEDY 90 est celle qui produit les meilleures prévisions, avec une médiane égale à 1 et une petite amplitude entre les centiles 10 et 90 (voir fig. 2), ce qui correspond à une petite dispersion ; donc, cette formule mène à la plus petite valeur de $S$ $\left(S=5.71 \times 10^{-3}\right)$. Le pourcentage des prévisions dont les erreurs sont inférieures à $50 \%$, c'est-à-dire, où $2 / 3<\mathrm{Ci} / \mathrm{Oi}<3 / 2(t a b) .6)$ est égal à $48 \%$ : le pourcentage des prévisions avec des erreurs inférieures à $100 \%$ $(1 / 2<\mathrm{Ci} / \mathrm{O}<<2)$ est de $67,5 \%(12,0+26,4+21,5+$ $7,6)$. Ces pourcentages sont assez élevés, ce qui indique une raisonnable concordance entre les valeurs calculées et observées, également révélée par le faible $(10,7 \%)$ pourcentage d'erreurs supérieures à $400 \%$.

Les formules de SHEN et Hung et de KARIM et KENNEDY 81 sont aussi très précises, avec des pourcentages élevés de prévisions dont les erreurs sont inférieures à $50 \%$ et à $100 \%$, et avec de faibles pourcentages de prévisions dont les erreurs sont supérieures à $400 \%$. Pourtant, les amplitudes des centiles 10 et 90 sont plus grandes que celle de KARIM et KENNEDY 90, ce qui se traduit par des valeurs de $S$ aussi supérieures.

Les formules de ENGELUND et HANSEN et de ACKERS et WHITE fournissent des résultats légèrement moins accep- 
Tableau 5. - Paramètres d'évaluation des formules pour les données du groupe 1.

\begin{tabular}{|c|c|c|c|c|c|c|}
\hline \multirow[b]{2}{*}{ Formule } & \multirow{2}{*}{$\begin{array}{c}\text { \% de données } \\
\text { couvertes }\end{array}$} & \multicolumn{3}{|c|}{ Centiles: } & \multirow{2}{*}{$\begin{array}{c}\% \text { de valeurs } \\
1 / 2<\mathrm{Ci} / \mathrm{Oi}<2\end{array}$} & \multirow[b]{2}{*}{$S\left(\times 10^{-3}\right)$} \\
\hline & & 10 & Médiane & 90 & & \\
\hline Engelun et Hansen & 87,0 & $1 / 1,9$ & 1,23 & 4,24 & 66,01 & 6,83 \\
\hline Graf & 99,7 & $1 / 2.2$ & 2,18 & 11,05 & 34,77 & 12,66 \\
\hline Shen et Hung & 99,8 & $1 / 3,4$ & 0,99 & 2,83 & 63,00 & 6,10 \\
\hline Ackers et White & 66,1 & $1 / 5,5$ & 0,96 & 2,39 & 59,46 & 9,69 \\
\hline Yang & 100,0 & $1 / 1,6$ & 2,63 & 360,32 & 35,59 & 28,56 \\
\hline Raju et al. & 94,0 & $1 / 6,3$ & 0,56 & 1,84 & 46,21 & 10,25 \\
\hline Brownlie & 96,0 & $1 / 4,0$ & 0,54 & 1,55 & 49,61 & 8,37 \\
\hline Karim et Kennedy 81 & 99,3 & $1 / 3,1$ & 0,79 & 3,03 & 60,92 & 6,36 \\
\hline Van Rijn & 44,9 & $1 / 23,5$ & 0,88 & 3,46 & 38,62 & 24,15 \\
\hline Karim et Kennedy 90 & 99,5 & $1 / 2,1$ & 1,00 & 3,42 & 67,63 & 5,71 \\
\hline
\end{tabular}

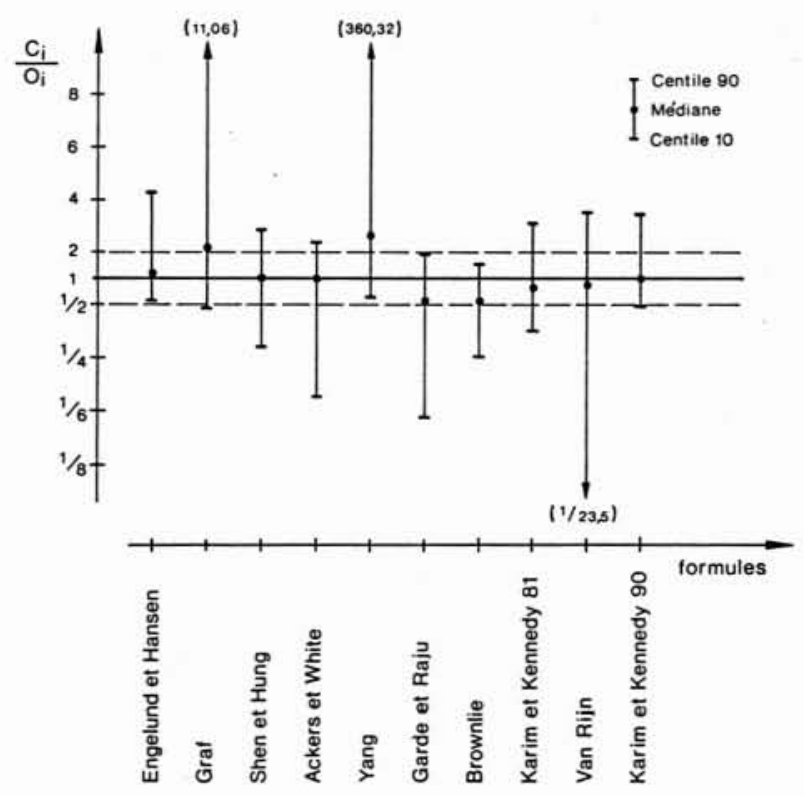

2. Valeurs de la moyenne et des centiles 10 et 90 de CilOi pour les données du Groupe 1. tables. Leurs pourcentages des prévisions dont les erreurs sont inférieures à $100 \%$ sont élevées mais elles présentent une large amplitude entre les centiles 10 et 90 . La formule de ENGELUND et HANSEN a tendance à surestimer les concentrations des sédiments tandis que la formule de ACKERS et WHITE a une tendance légèrement contraire. Appliquée au Brahmapoutre (Bangladesh), la formule de ENGELUND et HANSEN donne des transports solides qui sont environ deux fois inférieurs à la réalité (RAMETTE [17]), ce qui ne confirme pas nos résultats.

La formule de BROWNLIE, de petite dispersion, sousestime nettement la concentration ; ceci dérive de la petite valeur de la médiane $(0,54)$ ainsi que de celles des centiles $10(1 / 4,0)$ et $90(1,55)$. La valeur de $S$ est donc plus élevée que celle des formules déjà discutées.

Les formules de GRAF et de YANG surestiment très fortement la concentration, tandis que celle de RAJU et al. la sous-estiment aussi nettement.

Nos résultats ne sont pas entièrement d'accord avec les conclusions d'autres études récentes, notamment avec BAKKER et al. [3]. Selon ces auteurs, qui ont appliqué les formules de ENGElund et HANSEn, Ackers et White, KARIM et KENNEDY 81, YANG et VAN RIJN aux données des canaux ACOP, les trois meilleures formules seraient

Tableau 6. - Histogramme des fréquences de $\mathrm{Ci} / \mathrm{Oi}$ des trois meilleures formules pour le Groupe 1.

\begin{tabular}{|c|c|c|c|c|c|c|c|c|c|c|c|c|}
\hline Formule $\quad \mathrm{Ci} / \mathrm{Oi}$ & $<\frac{1}{8}$ & $\frac{1}{8}$ à $\frac{1}{6}$ & $\frac{1}{6} \grave{a} \frac{1}{4}$ & $\frac{1}{4} \mathrm{a} \frac{1}{2}$ & $\frac{1}{2} \grave{\mathrm{a}} \frac{2}{3}$ & $\frac{2}{3}$ à 1 & $1 \mathrm{a} \frac{3}{2}$ & $\frac{3}{2}$ à 2 & 2 à 4 & 4 à 6 & 6 à 8 & $>8$ \\
\hline Karim et Kennedy 90 & 0,9 & 0,5 & 1,0 & 9,1 & 12,0 & 26,4 & 21,5 & 7,6 & 12,6 & 5,0 & 1,6 & 1,7 \\
\hline Shen et Hung & 2,9 & 1,4 & 3,2 & 11,3 & 9,2 & 22,7 & 21,6 & 9,6 & 13,3 & 2,5 & 0,9 & 1,4 \\
\hline Karim et Kennedy 81 & 2,4 & 1,4 & 2,0 & 17,0 & 14,6 & 25,6 & 14,1 & 6,6 & 9,9 & 3,7 & 1,5 & 1,2 \\
\hline
\end{tabular}


celles de Engelund et HANSEn, ACKers et White et KARIM et KENNEDY 81 . Pourtant, ces résultats sont probablement moins significatifs que les nôtres parce que le nombre des données de BAKKER et al. [3] était assez réduit et leur qualité était aussi douteuse (voir BAKKER et al.. p. 11). Selon Raju etal. [7]. qui ont vérifié la validité des formules de ACKERS et WHITE. D’ENGELUND et HANSEN et de GRAF pour la plupart des données de Peterson et Howels [16], la formule de Ackers et WHITE serait assez précise, avec des erreurs inférieures à $50 \%$ dans $80 \%$ des cas. A part le nombre réduit de formules essayées par RAJU et al., il faut souligner que les données de Peterson et Howels avaient beaucoup d'erreurs qui ont été éliminées, plus tard, par le Delft Hydraulics Laboratory et par CARDOSO et BELo [6]. La conclusion de RAJU el al. perd donc une partie de sa signification. Il faut encore souligner qu'aucune des études connues n'a testé la formule de KARIM et KENNEDY 90 qui est, d'ailleurs, la plus précise pour les données du Groupe l.

\subsubsection{Données du Groupe 2}

Les résultats concernant le Groupe 2 (fleuves portugais) sont résumés dans le tableau 7, la figure 3 et le tableau 8 .

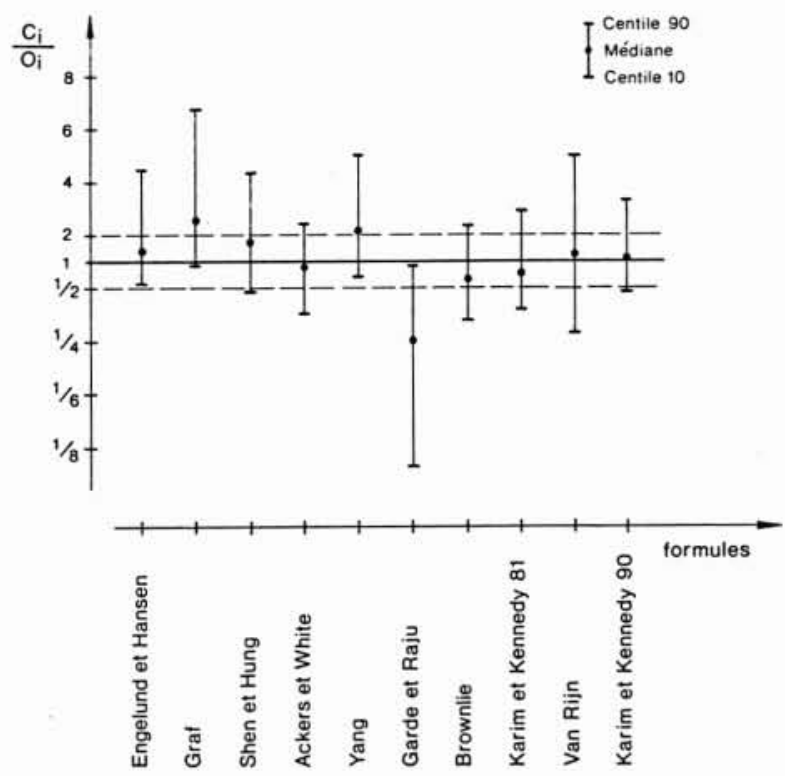

3. Valeurs de la moyenne et des centiles 10 et 90 de CilOi pour les données du Groupe 2.

Tableau 7. - Paramètres d'évaluation des formules pour les données du Groupe 2.

\begin{tabular}{|c|c|c|c|c|c|c|}
\hline \multirow[b]{2}{*}{ Formule } & \multirow{2}{*}{$\begin{array}{c}\text { \% de données } \\
\text { couvertes }\end{array}$} & \multicolumn{3}{|c|}{ Centiles: } & \multirow{2}{*}{$\begin{array}{c}\% \text { de valeurs } \\
1 / 2<\mathrm{Ci} / \mathrm{Oi}<2\end{array}$} & \multirow[b]{2}{*}{$S\left(x 10^{-3}\right)$} \\
\hline & & 10 & Médiane & 90 & & \\
\hline Engelund et Hansen & 100,0 & $1 / 1,7$ & 1,37 & 4,38 & 66,32 & 13,31 \\
\hline Graf & 100,0 & $1 / 1,2$ & 2,56 & 6,69 & 32,21 & 22,95 \\
\hline Shen et Hung & 100,0 & $1 / 2,3$ & 1,63 & 4,14 & 49,47 & 15,60 \\
\hline Ackers et White & 100,0 & $1 / 3,0$ & 0,83 & 2,46 & 60,63 & 12,06 \\
\hline Yang & 100,0 & $1 / 1,4$ & 2,08 & 4,97 & 40,21 & 19,02 \\
\hline Garde e Raju & 97,0 & $1 / 8,8$ & 0,25 & 0,83 & 17,97 & 31,67 \\
\hline Brownlie & 100,0 & $1 / 3,3$ & 0,67 & 2,37 & 55,79 & 13,75 \\
\hline Karim et Kennedy 81 & 100,0 & $1 / 2,8$ & 0,79 & 2,87 & 59,97 & 12,12 \\
\hline Van Rijn & 54,0 & $1 / 3,7$ & 1,35 & 4,98 & 45,49 & 24,09 \\
\hline Karim et Kennedy 90 & 100,0 & $1 / 2,1$ & 1,08 & 3,34 & 66,11 & 11,52 \\
\hline
\end{tabular}

Tableau 8. - Histogramme des fréquences de $\mathrm{Ci} / \mathrm{O} i$ des trois meilleures formules pour le Groupe 2.

\begin{tabular}{|c|c|c|c|c|c|c|c|c|c|c|c|c|}
\hline Formule $\quad \mathrm{Ci} / \mathrm{Oi}$ & $<\frac{1}{8}$ & $\frac{1}{8}$ à $\frac{1}{6}$ & $\frac{1}{6} \mathrm{a} \frac{1}{4}$ & $\frac{1}{4} \mathrm{a} \frac{1}{2}$ & $\frac{1}{2} \grave{a} \frac{2}{3}$ & $\frac{2}{3}$ à 1 & $1 \mathrm{a} \frac{3}{2}$ & $\frac{3}{2}$ à 2 & 2 à 4 & 4 à 6 & 6 à 8 & $>8$ \\
\hline Karim et Kennedy 90 & 0,6 & 0,4 & 0,6 & 9,3 & 13,1 & 22,8 & 30,2 & 10,1 & 14,7 & 4,0 & 1,3 & 2,9 \\
\hline Ackers et White & 1,3 & 0,2 & 1,7 & 20,6 & 13,9 & 20,6 & 16,0 & 10,1 & 11,8 & 1,7 & 0,8 & 1,3 \\
\hline Karim et Kennedy 81 & 1,3 & 0,8 & 1,1 & 20,4 & 15,3 & 21,9 & 15,0 & 7,5 & 9,9 & 3,2 & 0,8 & 2,7 \\
\hline
\end{tabular}


La différence la plus importante à signaler est le fait que la formule de ACKERS et WHITE apparaît entre les trois meilleures au détriment de la formule de SHEN et HUNG. Donc, les formules de KARIM et KENNEDY 90, de ACKERS et White et de KARIM et KENNEDY 81 sont celles qui produisent les meilleurs résultats pour ce groupe, avec des petites dispersions et des pourcentages élevés de prévisions dont les erreurs sont inférieures à $100 \%$ (pourcentages supérieurs à $60 \%$ ) ; les valeurs de $S$ sont toujours inférieures à $12,12 \times 10^{-3}$.

La formule de KARIM et KENNEDY 90 présente un pourcentage $(53 \%)$ de résultats dont les erreurs sont inférieures à $50 \%$, nettement mieux que la formule de ACKERS et WhITE $(37 \%)$ et que celle de KARIM et KENNEDY $81(37 \%)$. Cette dernière présente une tendance à sous-estimer la concentration des sédiments. La formule de ACKERS et WHITE est celle qui produit le pourcentage le plus faible $(7,1 \%)$ de prévisions dont les erreurs sont supérieures à $400 \% \quad(\mathrm{Ci} / \mathrm{O} i<1 / 4$;
$\mathrm{Ci} / \mathrm{O} i>4)$. Finalement, on doit signaler que les formules de BROWNLIE et de ENGELUND et HANSEN présentent un pourcentage élevé ( $>55 \%$ ) de résultats dont les erreurs sont inférieures à $100 \%$; pourtant, la formule de BROwNLIE présente une légère tendance à sous-estimer la valeur de la concentration des sédiments et celle de ENGELUND et HANSEN conduit à une dispersion non négligeable. Toutes les autres formules sont moins adéquates.

Les remarques faites dans le $\$ 4.2 .1$, pour les données du Groupe 1, à propos de la comparaison entre les résultats de cette étude et la littérature existante sont aussi valables pour les résultats concernant le Groupe 2 .

\subsection{Evaluation des formules par des sous-ensembles du plan $Z$, Fr}

Les résultats de l'étude pour les sous-ensembles $A_{2} A_{3}$. $A_{2} A_{4}, A_{3} A_{3}$ et $A_{3} A_{4}$ (voir tabl. 2) de chaque groupe de données sont résumés dans les tableaux 9 et 10 pour les

Tableau 9. - Meilleures formules pour les sous-ensembles du plan Z, Fr, Groupe 1.

\begin{tabular}{|c|c|c|c|c|}
\hline Sous-ensemble & Formules & $\begin{array}{l}\% \text { de données } \\
\text { couvertes }\end{array}$ & $\begin{array}{c}\% \text { de valeurs } \\
1 / 2<\mathrm{Ci} / \mathrm{Oi}<2\end{array}$ & $\begin{array}{c}S \\
\left(\times 10^{-3}\right)\end{array}$ \\
\hline $\begin{array}{c}A_{2} A_{3} \\
\text { (313 enregistrements) }\end{array}$ & $\begin{array}{l}\text { Karim et Kennedy } 90 \\
\text { Engelund et Hansen } \\
\text { Karim et Kennedy } 81\end{array}$ & $\begin{array}{l}100,0 \\
87,2 \\
100,0 \\
\end{array}$ & $\begin{array}{r}66,7 \\
72,35 \\
59,11 \\
\end{array}$ & $\begin{array}{l}14,50 \\
15,52 \\
15,54 \\
\end{array}$ \\
\hline $\begin{array}{c}\mathrm{A}_{2} \mathrm{~A}_{4} \\
\text { (335 enregistrements) }\end{array}$ & $\begin{array}{l}\text { Karim et Kennedy } 90 \\
\text { Brownlie } \\
\text { Karim et Kennedy } 81\end{array}$ & $\begin{array}{c}100,0 \\
90,1 \\
100,0\end{array}$ & $\begin{array}{l}53,73 \\
54,97 \\
50,45\end{array}$ & $\begin{array}{l}17,52 \\
17,72 \\
17,80\end{array}$ \\
\hline $\begin{array}{c}A_{3} A_{3} \\
\text { (966 enregistrements) }\end{array}$ & $\begin{array}{c}\text { Shen et Hung } \\
\text { Karim et Kennedy } 90 \\
\text { Karim et Kennedy } 81\end{array}$ & $\begin{array}{l}100,0 \\
99,2 \\
99,0 \\
\end{array}$ & $\begin{array}{l}77,33 \\
72,86 \\
66,42 \\
\end{array}$ & $\begin{array}{l}5,56 \\
6,80 \\
7,63 \\
\end{array}$ \\
\hline $\begin{array}{c}\mathrm{A}_{3} \mathrm{~A}_{4} \\
\text { (128 enregistrements) }\end{array}$ & $\begin{array}{c}\text { Karim et Kennedy } 90 \\
\text { Brownlie } \\
\text { Raju et al. } \\
\end{array}$ & $\begin{array}{l}100,0 \\
100,0 \\
82,8 \\
\end{array}$ & $\begin{array}{l}66,93 \\
45,31 \\
47,17\end{array}$ & $\begin{array}{l}24,96 \\
34,07 \\
35.21 \\
\end{array}$ \\
\hline
\end{tabular}

Tableau 10. - Meilleures formules pour les sous-ensembles du plan $Z, F r$, Groupe 2.

\begin{tabular}{|c|c|c|c|c|}
\hline Sous-ensemble & Formules & $\begin{array}{c}\% \text { de données } \\
\text { couvertes }\end{array}$ & $\begin{array}{c}\% \text { de valeurs } \\
1 / 2<\mathrm{Ci} / \mathrm{Oi}<2\end{array}$ & $\begin{array}{c}\mathrm{S} \\
\left(\times 10^{-3}\right)\end{array}$ \\
\hline $\mathrm{A}_{2} \mathrm{~A}_{3}$ & Engelund et Hansen & 100,0 & 87,56 & 11,14 \\
(201 enregistrements) & Karim et Kennedy 90 & 100,0 & 81,09 & 13,96 \\
& Karim et Kennedy 81 & 100,0 & 67,16 & 19,64 \\
\hline $\mathrm{A}_{2} \mathrm{~A}_{4}$ & Ackers et White & 100,0 & 61,32 & 16,65 \\
$(243$ enregistrements) & Brownlie & 100,0 & 62,14 & 17,58 \\
& Karim et Kennedy 81 & 100,0 & 51,85 & 21,15 \\
\hline $\mathrm{A}_{3} \mathrm{~A}_{3}$ & Karim et Kennedy 90 & 100,0 & 84,62 & 35,27 \\
& Engelund et Hansen & 100,0 & 92,13 & 36,07 \\
& Shen et Hung & 100,0 & 74,91 & 49,17 \\
\hline
\end{tabular}


trois meilleures formules. Ils ne diffèrent pas de façon significative des résultats globaux déjà présentés. Pour le Groupe 2, c'est-à-dire, pour les données des fleuves portugais, nous ne tenons pas compte du sous-ensemble $A_{3} A_{4}$ car seuls deux enregistrements y sont présents.

Les formules de KARIM et KENNEDY (81 et 90) se trouvent, à nouveau, pour les sous-ensembles, parmi les trois meilleures formules, se détachant clairement comme les plus adéquates. Les exceptions concernent les sousensembles $A_{3} A_{4}$ du Groupe $I$ et $A_{2} A_{4}$ et $A_{3} A_{3}$ du Groupe 2 où l'une des deux n'est pas présente.

La formule de ENGELUND et HANSEN est parmi les meilleures pour les sous-ensembles $A_{2} A_{3}$ du Groupe $l$ et $A_{2} A_{3}$ et $A_{3} A_{3}$ du Groupe 2 . La formule de BrownLIE est parmi les trois meilleures pour les sous-ensembles $A_{2} A_{4}$ et $A_{3} A_{4}$ du Groupe I et $A_{2} A_{4}$ du Groupe 2, c'est-àdire, pour des valeurs de $Z$ supérieures à 1000 . La formule de SHEN et HUNG produit de bons résultats pour le sous-ensemble $A_{3} A_{3}$ des Groupes I et 2. La formule de ACKERS et WHITE est parmi les meilleures seulement pour le sous-ensemble $A_{2} A_{4}$ du Groupe 2 tandis que celle de RaJu et al. l'est seulement pour $A_{3} A_{4}$ du Groupe 1 .

\section{Conclusions}

Dans cette étude nous avons évalué l'applicabilité de dix formules de transport solide total en comparant les résultats avec des données existantes qui couvrent des situations observées dans les fleuves Tejo et Mondego. Nous pouvons résumer les conclusions les plus importantes comme suit :

- Les formules de KARIM et KENNEDy (90 et 81 ) sont toujours parmi les trois meilleures quand on les applique à la totalité des données des Groupes 1 et 2 ; elles sont applicables à la plupart de ces données et produisent de petites erreurs.

- Les formules de SHEN et HUNG et de ACKers et WHITE produisent aussi de bonnes prévisions, respectivement, pour les Groupes 1 et 2.

- Les formules de Karim et KenNedy ( 90 et 81 ) sont encore parmi les meilleures pour la majorité des sousensembles des Groupes I et 2.

- Les formules de ENGELUND et HANSEN, de ACKERS et White, de Brownlie et de Shen et Hung produisent de bons résultats pour certains sous-ensembles, particulièrement pour les données du Groupe 2 (fleuves portugais).

Dans l'Annexe, nous présentons les formules les plus précises pour l'ensemble des données des Groupes $I$ et 2 , i.e., les formules de KARIM et KENNEDY 81 et 90 [11], la formule de ACKERS et WHITE [1] et la formule de SHEN et HUNG $|18|$.

Remerciements: C'est un devoir très agréable de remercier la Junta Nacional de Investigação Científica e Technológica pour la bourse attribuée au second auteur de l'article ; cette bourse a rendu possible la réalisation de son travail de maîtrise [13] (à l'Instituto Superior Técnico et au Laboratório Nacional de Engenharia Civil) sur lequel cet article est basé. Nous exprimons aussi notre reconnaissance à Mme Adriana Cardoso qui a apporté son soutien dans la préparation des codes de calcul de certaines des formules analysées.

\section{Bibliographie}

11] ACkers P., White R. (1973). - Sediment transport : new approach and analysis. Journal of the Hydraulics Division, ASCE, Vol. 99, $\mathrm{N}^{\circ} \mathrm{HY} 11$. Nov.

[2] ASCE (1975). - Sedimentation Engineering. Vol. N* 54. Ed. Vanoni V. A., New York.

[3] BAKKeR B., VermaAs H., ChOUdrI A.M. (1989). - Regime theories updated or outdated. Deft Hydraulics Publications, $N^{*} 416$, June.

[4] Bouvard M. (1983). - Ouvrages de dérivation et transports solides. La Houille Blanche $\mathrm{N}^{\text {os }} 3 / 4$.

[5] BRownLIE W.R. (1981). - Prediction of flow depth and sediment discharge in open channels. Rept. Kh-R-34A, W.M. Keck Laboratory of Hydraulics and Water Resources, California Institute of Technology, Pasadena, California.

[6] Cardoso A.H., Belo J.C. (1986). - Contribution to the compilation of alluvial channel data. Relatório, Laboratório Nacional de Engenharia Civil, Lisboa.

[7] RaJU K.R., GARDE R.J., BhardwaJ R.C. (1981). - Total load transport in alluvial channels. Journal of the Hydraulics Division, ASCE, Vol. 107, N HY 2, Fev.

[8] Gomez B., Church M. (1989), - An assessment of bed load sediment transport formulae for gravel bed rivers. Water Resources Research, Vol. 25, N*6, June.

[9] GRAF W.H. (1971), - Hydraulics of sediment transport. McGraw-Hill, New York.

[10] GraF W.H., CaO H.H., Bathurst J.H. (1984), - Blench equilibrium parameters: supplementary data. Journal of Hydraulic Engineering. ASCE, Vol. 110. N¹1, Nov.

[11] KARIM M.F., KENNEDY J.F. (1990), - Menu of couple velocity and sediment discharge relations for rivers. Journal of Hydraulic Engineering, Vol. 116, N*8, Aug.

[12] Murteira B.J. (1983), - Estatística Descritiva. McGraw-Hill de Portugal.

[13] NEVES M.G.O. (1992), - Escoamentos uniformes em canais de leito nuóvel (en Portugais). Tese de Mestrado em Hidráulica e Recursos Hídricos, Instituto Superior Técnico, Lisboa.

[14] Ol.tveira R.P., Cardoso A.H. (1990), - Resistência ao escoamento em leitos aluvionares : estudo comparativo de critérios existentes (en Portugais). Informação Técnica de Hidráulica, ITH 32, LNEC, Lisboa.

[15] Peterson A.W., Blench T. (1980), - Graphed equilibrium parameters of channels formed in sediment. Can. J. Civ. Eng., Vol. 7, National Research Council of Canada.

[16] Peterson A.W., Howels R.F. (1973). - A compendium of solids transport data for mobile boundary channels. Report N* HY-1973-ST3, Department of Civil Engineering, University of Alberta, Canada.

[17] RAmette M. (1993). - Communication personnelle.

[18] SHEN H.N. (1971), - Total sediment load. In « River Mechanics », H.N. Shen Ed., chapter 13, Fort Collins, Colorado.

[19] VAn Run L.C. (1984). - Sediment transport. Part I : bed load transport. Journal of Hydraulic Engineering, ASCE, Vol. $110, \mathrm{~N}^{\circ} 10$, Oct.

[20] VAN Run L.C. (1984). - Sediment transport. Part II : suspended load transport. Journal of Hydraulic Engineering. ASCE, Vol, $110, \mathrm{~N}^{*} 11$, Nov. 
[21] Weast R.C., Astle M.J. (1981). - CRC Handbook of Chemistry and Physics. CRC Press Inc., Florida, USA.

[22] White W.R., Millut H., Crabbe A.D. (1973). - Sediment transport: an appraisal of available methods. Report $\mathrm{N}^{\circ}$ INT 119, Hydraulics Research Station, Wallingford, England, Nov.

[23] YANG C.T. (1982). - Sediment transport and unit stream power function. Journal of the Hydraulics Division, ASCE, Vol. 108, N`HY 6, June.

\section{ANNEXE}

Les formules de transport solide total les plus performantes pour les données utilisées

\section{Formule de SHEN et Hung}

A l'aide de l'analyse de régression multiple appliquée aux données de 514 expériences en laboratoire et de 63 observations dans la nature, SHEN et HUNG en 1971 (voir SHEN [18]), ont proposé la formule suivante :

$$
\log C=a_{0}+a_{1} X+a_{2} X^{2}+a_{3} X^{3}
$$

où $C$ est la concentration du matériau du fond transporté, en poids (ppm), $X$ est un paramètre défini par :

$$
X^{(1 / 0,0050702)}=\frac{V J^{0,57}}{\omega^{0,32}}
$$

$V$ est la vitesse moyenne de l'écoulement (ft/sec), $J$ est la pente de la ligne d'énergie, $\omega$ est la vitesse de chute $(\mathrm{ft} / \mathrm{sec})$ d'une particule de diamètre égal au diamètre median des sédiments du fond, et les constantes $a_{0}$, $a_{1}, a_{2}$ et $a_{3}$ sont des constantes établies par régression comme $\quad a_{0}=-107404,46, \quad a_{1}=324214,75$, $a_{2}=-326309,59$ et $a_{3}=109503,87$.

\section{Formule de ACKERs et WhITE}

La formule proposée par ACKERS et WhITE, en 1973, pour le transport solide total peut s'écrire de la façon suivante :

$$
\left(\frac{u *}{V}\right)^{C_{1}} \frac{\gamma}{\gamma_{s}} \frac{h}{D_{50}} C=C_{2}\left(\frac{F_{1}}{C_{3}}-1\right)^{C_{4}}
$$

où :

$$
F_{1}=\left(\frac{u_{*}^{c_{1}}}{\sqrt{\frac{\gamma_{s}-\gamma}{\rho} D_{50}}}\right)\left(\frac{V}{\sqrt{32} \log \frac{10 h}{D_{50}}}\right)^{1-c_{1}} .
$$

Dans les équations (A.3) et (A.4), les coefficients $C_{1}, C_{2}, C_{3}$ et $C_{4}$ dépendent de la valeur du paramètre d.:

$$
d=\left(\frac{\gamma_{s}-\gamma}{\rho v^{2}}\right)^{\frac{1}{3}} D_{50} .
$$

Pour $d .>60,0, C_{1}=0,0, C_{2}=0,025, C_{3}=0,17$ et $C_{4}=1,50$. Dans l'intervalle $1,0 \leqslant d . \leqslant 60,0$,

$$
C_{1}=1,0-0,56 \log d \text {. }
$$

$\log C_{2}=2,86 \log d .-(\log d .)^{2}-3,53$

$$
\begin{aligned}
& C_{3}=\frac{0,23}{d^{1 / 2}+0,14} \\
& C_{4}=\frac{9,66}{d *+1,34} .
\end{aligned}
$$

Dans les équations (A.3) à (A.5), $u$. est la vitesse de frottement sur le fond, $\gamma$ est le poids volumique du fluide, $\gamma_{s}$ est le poids volumique du matériau du fond, $h$ est la profondeur de l'écoulement, $D_{50}$ est le diamètre médian du matériau du fond, $\rho$ est la masse volumique du fluide et $v$ est la viscosité cinématique du fluide.

\section{Formules de KARIM et KEnNEDY}

La première formule proposée, en 1981, par ces auteurs pour la prévision du transport solide total peut être écrite sous la forme :

$$
\begin{aligned}
& \log \left[\frac{q_{s}}{\sqrt{g(s-1) D_{50}^{3}}}\right]=-2,279+ \\
& +2,972 \log \left[\frac{V}{\sqrt{g(s-1) D_{50}}}\right] \\
& +1,060 \log \left[\frac{V}{\sqrt{g(s-1) D_{50}}}\right] \log \left[\frac{u \cdot-u_{*_{c}}}{\sqrt{g(s-1) D_{50}}}\right] \\
& +0,299 \log \left(\frac{h}{D_{50}}\right) \log \left[\frac{u \cdot-u_{*_{c}}}{\sqrt{g(s-1) D_{50}}}\right]
\end{aligned}
$$

où $q_{s}$ est le débit solide total, en volume, par unité de largeur, $g$ est l'accélération gravitationnelle, $s$ est la densité des sédiments et $u_{*_{c}}$ est la valeur critique de la vitesse de frottement sur le fond, définie sur la courbe de Shields.

Plus récemment, en 1990, KaRIM et KENNEDY ont suggéré une nouvelle formule de transport solide total, sous la forme :

$$
\begin{aligned}
\frac{q_{s}}{\sqrt{g(s-1) D_{50}^{3}}}=0,00151 & {\left[\frac{V}{\sqrt{g(s-1) D_{50}}}\right]^{3.369} \times } \\
\times & {\left[\frac{u \cdot-u_{v_{c}}}{\sqrt{g(s-1) D_{50}}}\right]^{0.840} }
\end{aligned}
$$

où la signification des variables a déjà été présentée avant. Selon KARIM et KenNEDY [11], l'équation (A.8) est pratiquement aussi précise que la formule (A.7) pour les écoulements bien au-dessus de la situation critique de début de mouvement et moins précise que (A.7) pour les écoulements avec des concentrations faibles de sédiments. 